\title{
Anabases
}

ANABASES Traditions et réceptions de l'Antiquité

$8 \mid 2008$

Varia

\section{Christopher STRAY (éd.), Gilbert Murray Reassessed.} Hellenism, Theater and International Politics

\section{Carlamaria Lucci}

\section{OpenEdition}

\section{Journals}

Edizione digitale

URL: http://journals.openedition.org/anabases/268

DOI: 10.4000/anabases.268

ISSN: 2256-9421

\section{Editore}

E.R.A.S.M.E.

\section{Edizione cartacea}

Data di pubblicazione: 1 ottobre 2008

Paginazione: 309-311

ISSN: 1774-4296

Notizia bibliografica digitale

Carlamaria Lucci, « Christopher stray (éd.), Gilbert Murray Reassessed. Hellenism, Theater and International Politics », Anabases [Online], 8| 2008, Messo online il 01 juillet 2011, consultato il 22 septembre 2020. URL : http://journals.openedition.org/anabases/268 ; DOI : https://doi.org/10.4000/ anabases. 268

Questo documento è stato generato automaticamente il 22 settembre 2020.

(c) Anabases 


\title{
Christopher STRAY (éd.), Gilbert Murray Reassessed. Hellenism, Theater and International Politics
}

\author{
Carlamaria Lucci
}

\section{NOTIZIA}

Christopher STRAY (éd.), Gilbert Murray Reassessed. Hellenism, Theater and International Politics, Oxford, New York, Oxford University Press, 2007, 400 p.

120 euros / ISBN 978-0-19-920879-1.

1 Il volume, edito da Christopher Stray, si compone di sedici contributi, la maggior parte dei quali presentati in occasione della conferenza su Gilbert Murray, organizzata presso l'Institute of Classical Studies di Londra nel luglio del 2005. Ciascuno dei testi ricostruisce un aspetto della vita dell'intellettuale britannico nato in Australia (1866-1957), tra quelli indicati come peculiari nel titolo della raccolta: rispettivamente l'attività di ellenista, la traduzione di testi teatrali dal greco e l'impegno politico nella Società delle Nazioni. L'introduzione, ad opera del curatore, sottolinea la necessità di una revisione critica che renda giustizia al carattere poliedrico di questo intellettuale, del quale sono state scritte finora biografie largamente insoddisfacenti (West 1984 e Wilson 1987).

2 Ad unire i contributi è l'individuazione, come chiave di lettura dell'intera opera di Murray, di una sintesi tra ellenismo e liberalismo, per cui i valori del mondo greco antico sarebbero stati assunti a paradigma di un'ispirazione liberale di matrice vittoriana. Questa opzione ideologica sarebbe alla base della professione di ellenista: $i$ saggi di Griffith e Collard mostrano come, ad una prima fase di attività filologica, culminata con l'edizione critica, di valore dibattuto, dei drammi euripidei (1902-08) segua, di pari passo con l'incardinamento accademico a Oxford (1905-08), il fiorire di un'attività divulgativa, mai abbandonata, più congeniale a quella vocazione di intellettuale impegnato che West, nel suo contributo, ascrive all'idealizzazione del 
modello paterno di Terence Aubrey. Nell'ambito dell'attività divulgativa, Griffith isola le monografie, di varia fortuna, dedicate al dramma greco: l'interpretazione storicoculturale, fornita da Murray, della produzione drammaturgica di Euripide (1913), di Aristofane (1933), di Eschilo (1940) farebbe leva sullo stabilimento di un'esplicita analogia tra l'Atene Periclea e l'Inghilterra riformista di epoca vittoriana, impegnata nella dominazione del mondo. Tali monografie presuppongono un'interpretazione ritualistica della religione greca, mutuata alle teorie di Frazer e della Harrison, che Griffith considera a buon diritto il suo contributo più duraturo agli studi ellenistici e la base per tendenze antropologiche recenti come quelle di Burkert e di Girard. Nella lettura di Parker, Murray rovescia tuttavia l'approccio primitivistico della Harrison in una direzione "umanistico-evolutiva", mirando a mettere in luce come, da una fase di totemismo originario, i Greci siano passati ad una fase religiosa evoluta, caratterizzata dal culto delle divinità olimpiche nel contesto istituzionalizzato della polis e dalla sublimazione degli istinti primari attraverso le creazioni estetiche dell'epica e della tragedia. Sarebbe questa l'idea-guida dei tre importanti contributi rispettivamente sulle origini dell'epica greca arcaica (The Rise of the Greek Epic 1907), sulle origini rituali della tragedia (appendice a Themis di J. H. Harrison, 1912), sulle fasi di sviluppo della religione greca (Four/Five Stages of Greek Religion 1912, 1925).

3 I saggi di Morwood e Macintosh focalizzano un ulteriore aspetto dell'attività divulgativa di Murray, intrecciata con la professione accademica, quella di apprezzato traduttore di drammi dal greco, per lo più di tragedie euripidee, destinate a pubblica lettura da parte di lui stesso, o all'adattamento teatrale per il grande pubblico, attraverso la cooperazione con registi e drammaturghi, tra i quali spicca l'amico G. B. Shaw. I saggi di Davies e McManus si concentrano su due opposte tipologie di relazioni intrecciate da Murray nell'ambiente accademico: quella, di sostanziale rivalità, con il collega latinista di Cambridge A. E. Housman, e quella di sostegno intellettuale e, soprattutto, di amicizia, con l'allievo e protetto J.A. K. Thomson, inseritosi tardivamente, proprio grazie a Murray, nel circuito accademico e oggi pressoché dimenticato.

4 Il passaggio al versante più propriamente politico dell'impegno di Murray si attua attraverso l'esame del cinquantennale rapporto epistolare (1901-1957) col filosofo della scienza Bertrand Russell su questioni inerenti la teoria e la pratica del liberalismo. L'analisi di Bruneau sottolinea come, a partire da una piattaforma di valori comuni, identificabili nello scetticismo religioso, nell'umanesimo e nell'internazionalismo, le posizioni dei due intellettuali si divarichino in merito alla politica internazionale. Al pacifismo radicale e allo scetticismo, manifestato da Russell, nei confronti degli organismi statuali e sovrastatuali, Bruneau oppone la propaganda di Murray a favore della prima guerra mondiale $\mathrm{e}$, in seguito, l'adesione alla causa della pace internazionale attraverso la partecipazione attiva alla fondazione della League of Nations (1918). I saggi di Ceadel e Wilson analizzano precisamente le implicazioni teoriche e pratiche, nonché i limiti dell'impegno internazionalista di Murray. Il progressivo scioglimento della Lega a partire dal 1936, sotto l'onda montante delle dittature nazi-fasciste, rivelerebbe l'intrinseca debolezza delle aspirazioni politiche dello stesso Murray : il suo liberalismo ellenizzante sarebbe la spia di un'impostazione idealistica, inadeguata a comprendere le reali dinamiche internazionali in un'epoca di totalitarismi e di corsa agli armamenti. Tale atteggiamento appare, agli occhi degli interpreti, l'indice di un sostanziale conformismo : pur vantando, negli anni giovanili, una militanza progressistica, Murray restò un convinto assertore dei diritti dell'impero 
britannico e, più tardi, dell'egemonia anglosassone nella politica mondiale. Proprio l'ambivalenza fra ricerca illuminata e accettazione dei valori tradizionali rende comprensibile, nell'interpretazione di Julia Stapleton, il rapporto di cooperazione fra Murray ed A.E. Zimmern, suo allievo ed epigono nella divulgazione dell'ellenismo, animato da valori patriarcali di stampo addirittura pre-liberale. I contributi, di Morris da una parte, di Bruneau e Wodell dall'altra, riprendono e sviluppano il filone riguardante l'attività divulgativa, condotta da Murray fino alla fine, concentrandosi rispettivamente sulla collaborazione radiofonica con la BBC, a partire dal 1925, e sulla corrispondenza con l'editore del Times a partire dal 1908. Il saggio di Lowe, relativo al poco noto interesse di Murray per la ricerca psichica, concretizzatosi in numerosi esperimenti di telepatia, conclude nel segno della problematicità un ritratto intellettuale rivelatosi tutt'altro che semplice e scevro di contraddizioni.

\section{AUTORI}

\section{CARLAMARIA LUCCI}

Dipartimento di Filologia Classica, Università di Pisa

carlamarialucci@interfree.it 